\title{
Open Source or Commercial Products for Electronic Data Capture in Clinical Trials? A Scorecard Comparison
}

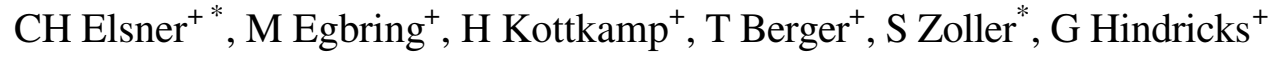 \\ ${ }^{+}$Heart Center Leipzig, Leipzig, Germany and ${ }^{*}$ HHL Business School Leipzig, Germany
}

\begin{abstract}
Collection and organization of data in Clinical Trials requires a sophisticated information management system. An ideal System should be easy accessible over webtechnology and should provide high security of data.

When deciding for a solution meanwhile even midand big-sized companies also evaluate Open-Source Platforms against pure Commercial Solutions as the use of Open-Source Products seems to get more and more feasible: Early Open-Source disadvantages like limited support and limited user-friendliness seem to disappear.

With this work the authors intended to discuss a basic scheme for which Software and Strategy to choose: Objective was to develop a Scorecard for decision support with parameters of the trial, user-needs, scale I dimension of the trial, financial budget, IT-ressources and long term objectives of the Companies IT-Strategy. The Scorecard can be obtained free from the authors.
\end{abstract}

\section{Introduction}

In Multicentre Studies the collection, organization and statistical analysis of studydata requires a sophisticated information support system. An ideal system should be easy accessible and should provide high security. For this reason Web-based or Web-enabled Remote-Data-Entry is seen as the most promising technology. This way of Data Management is ideal for data entry from distributed locations that have little or no computer infrastructure.

\subsection{Authors' experience and objective}

In the field of Data Collection for Clinical Trials the Authors have experience with OPEN-Source engineered products set up in a LAMP and/or PHP and ODBC environment [1], have used the PhOSCo ${ }^{\mathrm{TM}}$ [2] Platform and have used professional tools (Oracle Clinical®, SAS ${ }^{\circledR}$ and Business Objects $\left.{ }^{\circledR}\right)[3]$.

The objective of this work was to develop a Scorecard for the evaluation of any Clinical Trial Setting to determine which Software and Strategy to choose. For this target to achieve, especially the experience from the use of the Oracle Clinical ${ }^{\circledR} 4$ / SAS ${ }^{\circledR} 8.2$ and the use of the PhOSCo ${ }^{\text {TM }}$ Platform where compared and discussed.

\section{Actual situation: market shares}

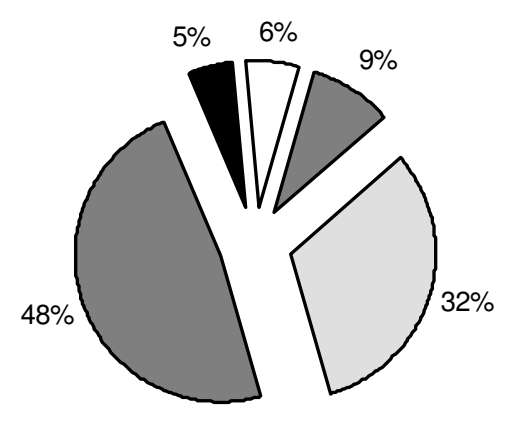

$\square$ ClinTrial
In-House
Solutions
$\square$ Other
Solutions
$\square$ In-House \&
Commercial
$\square$ Oracle Clinical

Figure 1. Market Shares for April 2002: Different Solutions for Clinical Trial Data Management [3]

A look into the market shares for April 2002 shows a high percentage for the commercial market leaders Phaseforward $\AA$ and Oracle $\AA$. But In-house and mixed inhouse and commercial solutions have already a share of $14 \%$ - these solutions are specially found in mid-size trials and not FDA-relevant clinical trials.

Analysing this 14\%-Share further it turns out that there is only one remarkable Open-Source Solution in use at this point of time: The $\mathrm{PhOSCo}^{\mathrm{TM}}$-Software (Predecessor is the Software ClinWare $\AA$ ). For this reason our discussion will focus on the comparison of $\mathrm{PhOSCo}^{\mathrm{TM}}$ with available Commercial Products.

\subsection{Procedure in scorecardsetup}

What are the most obstacles in Remote-Data Entry and what key requirements have to be evaluated for a Companies' Software Selection?

For the generation of the Scorecard the Keypoints from known projects [1] [2] [3] were collected and put in relation to the common variables of a clinical trial. Additionally the parameters of the Companies ITStrategy and IT Environment were put into equation for the scorecard. The resulting Questions were clustered into groups to determine a score for each single Keypoint and determine a score for the best recommendation. 


\section{Identification of key criteria}

The following Criteria were identified to be crucial in Setup of a Clinical Trial Data Management Environment:

\subsection{Process scalability and flexibility}

Depending on the Trial's scale and potential diversity of future Trials a Remote-Data-Entry solution should be more or less open from a process-oriented point of view. To identify the individual requirements, questions for Trial Settings with rapid changing requirements were clustered. For those it is crucial to be able to accommodate the software in different ways: fast performing tasks inhouse, easy working with partners, cooperation with other entitities (academia or other Pharma) or any other model representing any combination. In this point Open-Source Software seems to have a clear advantage [4].

\subsection{Technology integration}

From a technology point of view the scalability and flexibility has to be seen more from the GUI, the database end and the intercomponent communications.

The decision, which software to choose depends on a variety of factors of the in-house IT-infrastructure. How big is the amount of data being managed, what flexibility is needed in database-interconnectivity and in databasediversity? How do different solutions fit into the actual IT-Infrastructure of a company? Is there a need to have database-clusters and what Backupconcepts are used as standard? Also the PhOSCo ${ }^{\mathrm{TM}}$ Software is based on open standards like Java / SQL / XML with effective possibilities for integration with downstream applications, it may not always be feasible to choose.

As a bottom line choosing Open Source - especially $\mathrm{PhOSCo}^{\mathrm{TM}}$ from this point of view seems for example especially feasible, when You have to keep Your Trial Software in a changing long-term hybrid communication model with the possibility of online working.

\subsection{Rollout process}

A simple and easy rollout process seems to be crucial - adequate to trial dimension and complexity. For the Scorecard a Cluster of Questions was setup to determine those variables.

While the environment must be validatable, all other factors can't be bought at the expense of the need for a complex or lengthy installation process. In the actual $\mathrm{PhOSCo}^{\mathrm{TM}}$ V1.3 specially Installation Process and GUI are not ideal for less complex Data Entry Scenarios, where for example a normal javascripted webfrontend (also from FDAs view) is enough.

In a capsulated standard Clinical Trial Environment with data from very distributed locations benefits and savings from Database- and Softwarelicenses can be overweighted by costs for installations and distributions of - for example - preinstalled laptops.

\subsection{CRF-to-bedside time}

A crucial factor in fast-pacing Trial Environments is the easy internalisation of the development of eCRFs and shortest possible time to first patient visit, using existing clinical staff.

Depending on the Setting of Trial Form Distribution and Handling of Development and Version Management it is worth to score the Performance of the Solutions according to the Trial Setting. Commercial Solutions like ClinTrial ${ }^{\circledR}$ or Studybuilder ${ }^{\circledR}[5]$ offer convenient version management and different distribution of CRFs on Handhelds, WWW and Paperbased.

The PhOSCo ${ }^{\mathrm{TM}}$ Plattform also offers a quite convenient eCRF Management but no different distribution management per se. For the Scorecard Analysis Questions for the Processes around the specific Clinical Trial Settings were setup and pictured in 23 items.

Depending on Complexity of CRFs, Processes, Distribution, potential Version Management and People involved a Recommendation can be given. Overall the recommended solution must seek to reduce the number and complexity of tasks required together with the number of people involved.

\subsection{Provider dependency}

Avoiding a dependency on special companies seems to be a critical success factor in every project. The TradeOff having to do this is often the need for a special, capsulated, single and cheap solution and no local ITRessources. The danger of this dependency is in terms of risks regarding software delivery and future maintenance as well as the danger of limited capability to scale up to the requirements of the programme.

Especially for larger-scale trials international service partners must be readily available to complement the internal resources, and the business concept has to be open to allow the addition of further partners when required.

\subsection{Lifecycle and version management}

Depending on the Spectrum of Trials being managed and the IT-Strategy there are some remarkable advantages in the use of Open-Source Software. For example the need for a simple and preferably automated process for maintenance of trial customisation and the software itself during the lifecycle of a trial, together with the capability of normal business change control management.

The Questionaire includes for example questions on Importance of fast Internationalisation and multilingual capabilities. 


\subsection{Legal regulations}

The Scorecard was put up with a special sequence of questions determining the need for special regulations regarding legal aspects and Software validation in a special Trial Setting. It turns out that the use of software under the regulations of the GNU [6] [7] has some specialities which can be a benefit or disadvantage for a company - a decision mostly basing on a general strategy to take back the ownership of a product back in house.

One mayor obstacle in Open-Source - the timeconsuming process of Software-Validation seems to be solved with $\mathrm{PhOSCo}^{\mathrm{TM}}$, as the tools for automated validation give a wide perspective mostly not found in the available commercial products.

\section{Discussion and results}

How is actual Situation at Companies having to decide between the two different approaches? A trend is heading clearly to Open-Source Products being developed from a core-version to individual needs of a company.

Of course implementing such a system is a major challenge, according to Sylvia Collins, who did this for Novartis [8]. Strong leadership, change management and broad authority were required. Most importantly it needs to be pushed forward as a business development, not an IT development.

As described above based on the popular PhOSCo ${ }^{\mathrm{TM}}$ product, the system at Novartis is continually being developed. It is deployed on 3000 laptops worldwide and has been deployed on 150 trials within 18 months. Database licence savings alone have been over $\$ 50$ million US Dollars [8]. The configuration and deployment of the clinical report forms takes around 9 weeks but Novartis hopes to improve this considerably.

The $\mathrm{PhOSCo}^{\mathrm{TM}}$ product is available for purchase, on a per trial or corporate basis, and is supplied with full Java source code for customisation and integration. With Open-Source Packages like $\mathrm{PhOSCo}^{\mathrm{TM}}$ even huge trials can be managed at low cost for software licenses. Overall choosing a professional environment makes trial control and management in points of standard integration and CRF-Design more convenient but also much more costly and not in every case more flexible.

Specially for Companies heading to a global OpenSource Strategy and/or having to keep Your Trial Software in a changing long-term hybrid communication model, $\mathrm{PhOSCo}{ }^{\mathrm{TM}}$ seems a viable alternative.
The Scorecard resulting from our work can be obtained free from the authors and is a 26 page questionnaire with chapters as described in 3. of this Paper and clustered questions on: A.Needs in ProjectManagement and Remote-Work Components at Studysites, B.Determination of Study-Size, Volume of Data and complexity of CRFs, C.Determination of needed Software-Components and their Scalability, D.Determination of IT-Knowledge, Ressources and Future Strategy for the Project, E.Situation and special Needs in Hardware and Security.

The Scorecard System allows to determine a Product Recommendation and weights the different portions visually. The Questionaire may be useful for other groups e.g. for determination which environment may be feasible for their single study approach, but also for project planning and further development of the questionnaire.

\section{References}

[1] M. Egbring, G. Hindricks, H. Kottkamp, Ch. Elsner. A Real-Time Monitoring Tool for Multicentre Studies in Cardiology. In: Journal of Computers in Cardiology Volume 2000; 27: 183-184.

[2] See URL http://www.phosco.de

[3] M. Egbring, J. Pölitz, Ch. Elsner. Experiences with a combination of Oracle Clinical ${ }^{\circledR} 4$ / SAS ${ }^{\circledR} 8.2$ / MedDRA ${ }^{\circledR} 4$ and Business Objects ${ }^{\circledR}$ 4. In: Proceedings of MedNet 2002, 7th World Conference on the Internet in Medicine, Amsterdam 2003; 1:57-59.

[4] T. H. Müller, M. Linzbach, S. Haseloff, B. Troschke. Einbindung eines Web-basierten Randomisierungsdienstes in die Open-Source Studiensoftware $\mathrm{PhOSCo}^{\mathrm{TM}}$. In: Informatik, Biometrie und Epidemiologie in Med. u. Biol. 2003; 34/3:376-378.

[5] See URL http://www.studybuilder.com

[6] See URL http://www.gnu.org

[7] Ch. Elsner, M.Berli. Workshop and Tutorial on the Use of Open-Source Software in HealthCare Applications. In: Proceedings of MedNet 2002, 7th World Conference on the Internet in Medicine, Amsterdam 2003; 1:12-13.

[8] Malorye Branca. Who's holding up EDC implementation? In: Bio-IT World (online), Bio-IT Ressource, 2002 URL www.bio-itworld.com/news/020503_report1928.html

Address for correspondence.

Christian Elsner

Thomasiusstr. 9

04109 Leipzig

Germany

Ch.Elsner@gmx.de 